\title{
LIST OF PARTICIPANTS
}

BADER, H.

BAKER, R. W.

Bentley, C. R.

Bernas, V. V.

Bertie, J. E.

Bilgram, J. H.

BLOCH, M. R.

BONeD, C.

Budd, W. F.

Bullemer, B.

Buser, $O$.

CAMP, $\mathbf{P}$.

Camplin, G. G.

Clausse, H.

Colbeck, S. C. Cooper, L. H. N. Couture, L.

Davidson, D. W.

Dorner, B.

Duval, $\mathbf{P}$.

EldRUP, M.

EngelhardT, $\mathbf{H}$.

Faure, P.

Glen, J. W.

Goodman, D. J.

Gribion, P. W. F.
Apartment 17 G, 2451 Bricknell Avenue, Miami, FL 33129, U.S.A. Department of Geology, LaFayette College, Easton, PA 18042, U.S.A.

Geophysical and Polar Research Center, 1215 W. Dayton Street, Madison, WI 53706, U.S.A.

Laboratoire Chimie-Physique, Université Paris XI, F-91405 Orsay, France.

Department of Chemistry, University of Alberta, Edmonton, Alberta T6G $2 \mathrm{G}_{2}$, Canada.

Laboratorium für Festkörperphysik, Eidg. Technische Hochschule Zürich, CH-8og3 Zürich, Switzerland.

Max-Planck Institut für Kernphysik, 69 Heidelberg 1 , Postfach 103980, Saupfercheckweg 1, Germany.

Laboratoire de Thermodynamique, Université de Pau et des Pays de l'Adour, F-64000 Pau, France.

Antarctic Division, Department of Science, Melbourne University, 568 St. Kilda Road, Melbourne, Victoria 3052, Australia.

Hochschule der Bundeswehr München, D-80r4 Neubiberg, Federal Republic of Germany.

Eidg. Institut für Schnee- und Lawinenforschung, $\mathrm{CH}-7260$ Weissfluhjoch, Davos, Switzerland.

Department of Physics, University of Maine, Orono, ME 04473, U.S.A.

Department of Physics, Handsworth Grammar School, Grove Lane, Birmingham B21 9ET, England.

Laboratoire de Thermodynamique, Université de Pau et des Pays de l'Adour, F-64000, Pau, France.

U.S. Army CRREL, Hanover, NH 03755, U.S.A.

2 Queens Gate Villas, Lipson, Plymouth PL4 7 PM, England.

Université Paris VI, 35 rue Alain Chartier, F-7501 5 Paris, France.

Division of Chemistry, National Research Council, Ottawa, Ontario KIA oR9, Canada.

Institut Max Von Laue-Paul Langevin, avenue des Martyrs, F-38042 Grenoble-cedex, France.

Laboratoire de Glaciologie, 2 rue Très-Cloîtres, F-38o3 r Grenoblecedex, France.

Chemistry Department, Risø, National Laboratory, DK-400o Roskilde, Denmark.

Division of Geological and Planetary Sciences, California Institute of Technology, Pasadena, CA 91125 , U.S.A.

Laboratoire de Spectrométrie Raman-Laser, Centre Universitaire de Savoie, B.P. 143, F-730 I Chambery-cedex, France.

Department of Physics, University of Birmingham, P.O. Box 363 , Birmingham Br 5 2TT, England.

Physics and Chemistry of Solids Group, Gavendish Laboratory, Madingley Road, Cambridge $\mathrm{CB}_{3}$ oHE, England.

Physics Department, University of St. Andrews, St. Andrews, Fife KY 16 gAL, Scotland. 
Io

Gross, G. W.

HaLe, B. N.

Halletr, J.

Hammer, C.

Hanley, T. O'D.

HIGASHI, A.

HOLDER, J.

Homer, D. R.

HoNDOH, T.

Hubmann, M.

ITAGAKI, $\mathbf{K}$.

JACGARD, C.

Jezek, K.

JOHARI, G. P.

JoHnson, D. M.

JoNES, S. J.

KAMB, B.

KASSNER, J. L.

KENNEDY, J. M.

KLINGER, J.

KLUG, D. D.

KOROTKEVICH, Y. S.

Kuво, Y.

LEVI, L.

LILE, R. C.

Lister, $\mathrm{H}$.

Lozowski, E.

Mackenzie, A. P.

Maeno, N.
JOURNAL OF GLAGIOLOGY

New Mexico Institute of Mines and Technology, Socorro, NM 8790I, U.S.A.

Cloud Physics Research Center, University of Missouri-Rolla, Rolla, MO 65401, U.S.A.

Desert Research Institute, Reno, NV 89507, U.S.A.

Geophysical Isotope Laboratory, Københavns Universitet, Haraldsgade 6, DK-2220 København N, Denmark.

Campion College, University of Regina, Regina $\mathrm{S}_{4} \mathrm{~S}_{0} \mathrm{~A}_{2}$, Canada.

Department of Applied Physics, Hokkaido University, Sapporo o6o, Japan.

Institut für allgemeine Metallkunde und Metallphysik an der Rhein-Westf. Technishen Hochschule Aachen, Kopernikusstrasse 14, Aachen, Federal Republic of Germany.

43 Lutley Drive, Pedmore, Stourbridge, West Midlands DY 9 oYQ. England.

Department of Applied Physics, Hokkaido University, Sapporo. o6o, Japan.

Technisch-Chemisches Laboratorium Eidg. Technische Hochschule Zürich, Universitätstrasse 6, CH-8092 Zürich, Switzerland.

U.S. Army CRREL, Hanover, NH 03755 , U.S.A.

Institut de Physique, Université de Neuchâtel, rue A.-L. Breguet I, CH 2000 Neuchâtel, Switzerland.

1215 W. Dayton Street, Madison, WI 53706, U.S.A.

Glaciology Division, Environment Canada, Ottawa, Ontario$\mathrm{KIA} \mathrm{OE}_{7}$, Canada.

60 Vivian Avenue, Wembley, Middlesex HA9 6RG, England.

Glaciology Division, Inland Waters Directorate, Environment Canada, Ottawa, Ontario $\mathrm{K}_{\mathrm{AA}} \mathrm{oE}_{7}$, Canada.

Division of Geological and Planetary Sciences, Galifornia Institute of Technology, Pasadena, CA gr125, U.S.A.

University of Missouri-Rolla, Rolla, MO 65401, U.S.A.

European Research Office, 223 Old Marylebone Road, London: NWI 5 TH, England.

Laboratoire de Glaciologic, 2 rue Très-Cloîtres, F-78031 Grenoblecedex, France.

National Research Council, Ottawa, Ontario KIA oRg, Canada. Arctic and Antarctic Institute, Leningrad, U.S.S.R.

Japan Bridge and Structure Institute, Zenkaren Building, 5-chome, Minami-Aoyama, Minato-ku, Tokyo, Japan.

Giudad de la Paz 2580, Buenos Aires, Argentina.

Meteorology Department, University of Melbourne, Parkville, Victoria 3052 , Australia.

Department of Geography, University of Newcastle-upon-Tyne, Newcastle-upon-Tyne NEi ${ }_{7} R U$, England.

Division of Mechanical Engineering, National Research Council, Ottawa, Ontario KIA oR6, Canada.

Department of Bioengineering, University of Washington, Seattle, WA 98195 , U.S.A.

Institute of Low Temperature Science, Hokkaido University, Sapporo 060, Japan. 
Maİ, C.

Matsuda, M.

Mazzega, E

Mizuno, Y.

Mogensen, O. E.

NAGLE, J. F.

NoLl, G.

OTto, R. V.

Paren, J. G.

Paterson, W. S、B.

Pedersen, O. B.

Pennino, U. Del

Perez, J.

Plummer, P. L. M.

Podzimek, J.

Prince, R. H.

Prodr, F.

Quervain, M. DE

Rrae, S. A.

RILEY, N. F.

Ross, R. G.

SHMAOKa, K.

SINHA, N. K

Stauffer, B.

Swithinbank, C. W. M. British Antarctic Survey, Madingley Road, Cambridge GB3 oET, England.

TAUBENBERGer, R.

Tusrma, $\mathrm{K}$.

Institut National des Sciences Appliqués, Bât. 502 Physique, 20 avenue Albert Einstein, F-69621 Villeurbanne-cedex, France.

Institute of Low Temperature Science, Hokkaido University, Sapporo o6o, Japan.

Istituto di Fisica della Università, 4 I roo Modena, Italy.

Institute of Low Temperature Science, Hokkaido University, Sapporo o6o, Japan.

Chemistry Department, Risø National Laboratory, DK-4000 Roskilde, Denmark.

Department of Physics, Carnegie-Mellon University, Pittsburgh, PA 15213 , U.S.A.

Department of Physics, University of Birmingham, P.O. Box 363 , Birmingham Br 5 2TT, England.

Pembroke College, Cambridge, England.

British Antarctic Survey, Madingley Road, Cambridge $\mathrm{CB}_{3}$ oET, England.

Department of Energy, Mines and Resources, 4th Floor, 880 Wellington Street, Ottawa, Ontario $\mathrm{K}_{1} \mathrm{~A}$ oE4, Canada.

Metallurgy Department, Risø National Laboratory, DK-400o Roskilde, Denmark.

Instituto di Fisica della Università, 41 I0o Modena, Italy.

Institut National des Sciences Appliqués, Bât. 502 Physique, 20 avenue Albert Einstein, F-69621 Villeurbanne-cedex, France.

Cloud Physics Research Center, University of Missouri-Rolla, Rolla, MO 6540r, U.S.A.

University of Missouri-Rolla, Rolla, MO 6540 I, U.S.A.

Department of Physics, York University, Toronto, Ontario $\mathrm{M}_{3} \mathrm{~J}$ i $\mathbf{P}_{3}$, Canada.

IFA-GNR, Bologna, Italy.

Eidg. Institut für Schnee- und Lawinenforschung, $\mathrm{CH}-7260$ Weissfluhjoch, Davos, Switzerland.

James Franck Institute, University of Chicago, 5640 Ellis Avenue, Chicago, IL 6o637, U.S.A.

Department of Geography, University of Newcastle-upon-Tyne, Newcastle-upon-Tyne NEI 7 RU, England.

School of Mathematics and Physics, University of East Anglia, Norwich NR4 7 TJ, England.

Physics Department, Ritsumeikan University, Kita-ku, Kyoto 603, Japan.

Division of Building Research, National Research Council of Canada, Ottawa, Ontario KIA oR6, Canada.

Physikalisches Institut, Universität Bern, Sidlerstrasse 5, CH-3or 2 Bern, Switzerland.

Laboratorium für Festkörperphysik, Eidg. Technische Hochschule Zürich, CH-8093 Zürich, Switzerland.

Institute of Low Temperature Science, Hokkaido University, Sapporo o6o, Japan. 
12

VALI, G.

VAssoille, $R$.

VERBERNE, J. B.

WALFORD, M. E. R.

Watanabe, $\mathrm{Z}$.

WeickManN, H. $\mathrm{K}$.

WHALLEY, E.

WHITWORTH, R. W.

WiLson, C. J. L.

Woon, P.
JOURNAL OF GLACIOLOGY

Department of Atmospheric Sciences, University of Wyoming, P.O. Box 3038, Laramie, WY 82071 , U.S.A.

Institut National des Sciences Appliqués, Bât. 502 Physique, 20 Avenue Albert Einstein, F-69621 Villeurbanne-cedex, France.

Fleerde 413, Amsterdam, Netherlands.

H. H. Wills Physics Laboratory, Tyndall Avenue, Bristol BS8 ITL, England.

Department of Physics, Fukushima University, Fukushima City 960 , Japan.

Atmospheric Physics and Chemistry Laboratory, NOAA Environmental Research Laboratories, Boulder, CO 80302, U.S.A.

National Research Council, Ottawa, Ontario KIA oR9, Canada. Department of Physics, University of Birmingham, P.O. Box 363 , Birmingham Bi 5 2TT, England.

School of Earth Sciences, University of Melbourne, Victoria 3052, Australia.

Box 44, Cross River, NY 105 18, U.S.A. 